\title{
Osteosarcoma after the age of fifty: a clinicopathological study
}

\author{
Christine Tempelaere ${ }^{\mathrm{a}}$, David Biau ${ }^{\mathrm{a}}$, Antoine Babinet ${ }^{\mathrm{a}}$, Philippe Anract $^{\mathrm{a}}$ \\ a Service d'orthopédie, Hôpital Cochin, AP-HP, Paris, France, Université Paris Descartes, \\ Paris, France
}

Corresponding author: Dr Christine Tempelaere, chris.tempelaere@gmail.com

\begin{abstract}
Introduction: Osteosarcoma, a primary malignant bone tumor, has a well-recognised double peak of incidence in early adolescence and after 50 years. This study investigates the clinical features and prognostic factors of patients older than 50 years with osteosarcoma.

Materiel and methods: From January 2000 to December 2012, in one bone tumor reference center, 32 patients aged more than 50 years at the diagnosis (mean age: 62.4 years (50-85), sex ratio: 13 males, 19 females) diagnosed with osteosarcoma were included. Patients younger than 50 years at diagnosis or with a non-histologically proved osteosarcoma were excluded. For each patient, we registered medical history, tumor location, systemic and local extension, treatment, and survival.

Results: $62 \%$ were located in the extremities and $28 \%$ in the axial skeleton. 6 were secondary sarcomas. Mean delay between first symptoms and biopsy was 7.4 months (range from 0 to 28 months). Ten patients had a systemic osteosarcoma with one or more pulmonary metastases. Six patients were treated with palliative care (18.8\%). Eighteen patients received neodajuvant chemotherapy, sixteen of them received postoperative chemotherapy. Twentyfive patients had surgery. Postoperative complications were reported in eight cases (25\%). Overall survival for all 31 patients was $25 \%$ at 5 years and $6.2 \%$ at 10 years. Survival without metastases was $15.6 \%$ at 5 years and nil at 10 years. Median survival time for the 22 localised osteosarcoma patients was 4 years $(0.9-12.6)$ versus 1.2 years $(0.3-12.3)$ for the 10 systemic osteosarcoma patients $(\mathrm{p}=0.01)$.
\end{abstract}

Conclusion: Metastases at diagnosis, age, axial location are worse prognostic for survival.

Key words: osteosarcoma, age 50 years, chemotherapy 


\section{Introduction}

Osteosarcoma, a primary malignant bone tumor, has a well-recognised double peak of incidence in early adolescence (75\%) and elderly in the seventh and eigth decades (20\%)[14]. Global incidence is 3 cases per million per year [5,6]. This primitive osseous tumor is principally localised on long bones metaphysis (90\%) [5].

During first part of the twentieth century, five-year survival was less than $20 \%$ with only one treatment, amputation. The deceases were due to pulmonary metastases [7,8]. After 1980s, neoadjuvant chemotherapy and surgical resection led to a ten-year survival rate of 60 to $70 \%$ for localised osteosarcoma. Whereas metastatic osteosarcoma patient's five-year survival remains 25 to $30 \%$ [9].

As a result of the rapid population aging, osteosarcoma has increased in the elderly $[2,10]$. Balducci et al.[11] assessed that in 2030, $70 \%$ of all cancer would happen in patients aged more than 65 years. Some authors concluded that the older the patient is, the less his survival rate is [12-17]; whereas others found no correlation between age and survival [18-20]. While a standard treatment is well defined for young patients, no standard strategy has been established for the older [21-24]. The efficacy of chemotherapy in elderly is still controversial [3,14,25-29].

Clinical and histopathological features of osteosarcoma in adolescent patient have been well described, but there are only a few reports of the clinical and radiographical features of osteosarcoma in elderly patients. Publications [2,3,14,26-31] indicated that most osteosarcoma in patients over the age of 50 years are secondary lesions.

The aim of this study was to investigate the clinical features and prognostic factors of a heterogeneous group of patients older than 50 years with osteosarcoma.

\section{Materiel and Methods}

This is a retrospective study carried out in one bone tumor specialised center. From January 2000 to December 2012, we included all patients with an osteosarcoma aged more than 50 years at diagnosis. Patients younger than 50 years at diagnosis or with a non-histologically proved diagnostic were excluded.

For each patient, we registered in the medical history a Paget's disease or a previous irradiation, the discovery mode (pain, mass or fracture), the time between initial symptoms and biopsy, the biopsy type (Tru-cut巴 $[32,33]$ or surgical), the tumor location and his systemic and local extension with Broders'histology grade [34] and general check-up. The treatment was also reported : chemotherapy, surgery with histological margin and tumor necrosis percentage post chemotherapy grade [19], radiotherapy and complications. At last follow-up, surviving patients were accounted for. 


\section{Statistics}

Differences between groups were assessed using Chi-squared test. Overall and disease free survival was calculated using Kaplan-Meier product limit method and the impact of prognostic factors was assessed using a log-rank test univariate analysis. A p-value $<0.05$ was considered significant. Overall survival was calculated from date of diagnosis until death or the last follow-up visit. Disease free survival was defined as the period until the local or systemic disease recurred. Statistical analysis was done with XLSTAT version 2014 (Addinsoft). Confidence intervals (CI) of $95 \%$ were calculated for the statistical parameters.

\section{Results}

From January 2000 to December 2012, 32 patients aged more than 50 years were histologically diagnosed with an osteosarcoma (Table 1). Mean age was 62.4 years (range from 50 to 85). There were 13 males and 19 females. The most common location in the extremities was the distal femur in 11 patients, followed by proximal femur in 6 , proximal or distal tibia, proximal humerus in 3 and calcaneus in one. In the axial bones, 5 patients had tumor in the pelvis, 2 in the scapula. In 27 cases, a soft tissue extension was noted. There were 6 patients with secondary osteosarcoma, 5 of which were associated with radiotherapy, one with pelvis Paget's disease. Diagnosis was discovered after pain in 22 patients, an increasing size mass in 6 patients, a traumatism without fracture in one patient and a pathologic fracture in one patient.

Mean delay between first symptoms and biopsy was 7.4 month (range from 0 to 28 months) except for one patient with a painful multiple year evolution. Biopsy was realised with a Trucut ${ }^{\circledR}$ in 6 patients and surgically in 26 patients. Broders' grade histology was II for 3 patients and III for 29 patients (Table 2). Ten patients had a systemic osteosarcoma with one or more pulmonary metastases. One patient had an extensive pelvis osteosarcoma with a palliative treatment and had no thoracic scanner. Twenty-one patients had a localised osteosarcoma. An external whole-body bone scan was done for 15 patients without other location. For the 17 other patients, no scintigraphy was found in the medical report.

One patient was lost just after the diagnosis.

Palliative care was decided in multidisciplinary staff meeting for six patients with a high grade pelvis osteosarcoma $(18.8 \%)$.

Fifteen patients underwent the recommended therapy which included neodajuvant chemotherapy, surgery and adjuvant chemotherapy. Eighteen patients received neodajuvant chemotherapy with a unique protocol for all patients: API-AI (doxorubicine/cisplatins/ifosfamide; doxorubicine/ifosfamide). Only the number of courses varied. Twenty-five patients received surgery: five underwent amputation and the twenty others received limb-salvage surgery. In all patients, the status of surgical margin was wide R0 with a circumferential resection margin inferior to $1 \mathrm{~mm}$ in 4 cases. Among the eighteen patients who received neoadjuvant chemotherapy, there was seven good responders with tumoral necrosis > to 90\% (grade III and IV[19]), ten bad responders and one deceased 
before surgery. The conservative surgery was resection without reconstruction (acromion, scapula, collarbone) for three patients, resection and reconstruction by massive prosthesis for fourteen, one resection and spacer, two resection and reconstruction by allograft and osteosynthesis. Sixteen of these eighteen patients received postoperative chemotherapy adapted to histologic response, chemotherapy tolerance and pulmonary extension.

For one patient, therapy was surgery with an amputation and adjuvant chemotherapy. Surgical biopsy concluded to an unclassifiable osteosarcoma. Diagnosis was done after amputation.

Six patients had only surgery ( 2 amputations and 4 limb-salvage surgeries) without chemotherapy.

Postoperative complications were reported in seven cases (28\%). Complications were infection treated surgically and with antibiotics in four cases (one hip arthroplasty infection at 8 days, one knee prosthesis infection at 12 days, and 2 scar infections at 1 month), multiples metastasis in the scar at 19 months in one case, and one massive knee prosthesis mechanical complication which needed surgery at 2 months in the last case.

At diagnosis, osteosarcoma was systemic with pulmonary metastases in 10 patients. Among these patients, two underwent pulmonary surgery with adjuvant chemotherapy, two only had adjuvant chemotherapy, five were on palliative care and one was lost.

Secondary metastases happened in thirteen patients with a mean of 29.7 months (range of 3 to 96): 10 pulmonary and 3 multiples bone locations. Patients with pulmonary metastases were treated surgically and with adjuvant chemotherapy in 4 cases, only with chemotherapy in 2 cases, and with palliative care in 4 cases. Multiples bone metastases were treated by palliative radiotherapy.

Six patients developed local recurrence after surgery with a mean delay of 11.7 months (range of 4 to 24). Recurrence surgical resection was made in 5 cases followed by local irradiation in 2 cases. Palliative care was decided for one patient with well-oriented analgesia.

At last follow-up (Table 3), eight patients were alive and in remission without systemic manifestations. Two patients were lost: one just after the diagnosis due to a treatment refusal and one at three years postoperative disease free due to moving. Overall survival (Figure 1) for all 31 patients was $25 \%$ at 5 years and $6.2 \%$ at 10 years. Survival without metastases was $15.6 \%$ at 5 years and nil at 10 years. Median survival time for the 31 patients was 3.3 years (range from 0.3 to 12.6). Median survival time for the 22 localised osteosarcoma patients (Figure 2) was 4 years $(0.9-12.6)$ versus 1.2 years $(0.3-12.3)$ for the 11 systemic osteosarcoma patients (Figure 3$)(\mathrm{p}=0.01)$. Median survival delay for living patients at the last follow-up was 4 years (3-12.6).

Survival was different depending on the initial symptoms: if it was a pathologic fracture, median survival was 3.7 years (1.5-5.4); if it was a mass, median survival was 5.8 years (1.2$12.6)$; if it was pain, and median survival was 2.5 years $(0.3-12.3)$. No significant difference 
was found between these different groups (pain versus mass: $p=0.08$; pain versus fracture: $\mathrm{p}=0.6)$.

Location was associated with a different prognosis. Median survival of the 9 axial osteosarcoma patients was 1 year (0.3-8.5) and for the 23 extremities osteosarcoma patients, it was 4 years $(0.9-12.6)(\mathrm{p}=0.02)$.

No significant difference was observed between amputation and limb-salvage surgery in terms of survival.

Median survival for the twelve patients who received chemotherapy was 4 years $(0.3-12.6)$ versus 1.5 years $(0.7-8.5)$ for the eleven patients with a treatment without chemotherapy $(\mathrm{p}=0.03)$.

\section{Discussion}

In the present study, we reviewed 32 osteosarcoma patients older than 50 years treated in one bone cancer centre to demonstrate clinical features and prognostic factors. The site of primary lesions, metastases at diagnosis, definitive surgery and chemotherapy were significant prognostic factors.

Osteosarcoma over the age of 50 years is not that uncommon (approximately 13\% of patients with osteosarcoma over the age of 50 years) [35]. Age is a well-known bad prognosis factor. Song and al.[36] compared survival in two groups: inferior or superior to 40 years. Five year survival without metastases was $40.1 \%$ in the older group and $61.5 \%$ in the younger patients. In our study, 5-year survival was $25 \%$, similar to other studies [3,13,14,27,37]. Elderly patients seem to wait a longer time between first symptoms and first medical advice in comparison with younger patients. Time between symptoms and diagnosis was shown to be higher in the elderly by Longhi and al.[27] who reported 4 months (0-73) for elderly and 10 weeks for adolescents. This is also observed in our study with a mean waiting time of 7.4 months (0-28).

In young patients, extremities are the most frequent locations. It decreases when the patient is older [12,37]. The incidence of axial osteosarcoma in elderly patients has been reported as $13.7-49 \%[2,3,13,14,26,27,27,29]$. In the present study, $31.2 \%$ patients had a tumor an axial site. Their survival was 2.3 years (0.3-8.5), significantly different overall survival of the 22 patients with an extremities location, which was 4.2 years $(0.9-12.6)(\mathrm{p}=0.02)$. This result confirms the previous articles about the severity of axial location.

In elderly, osteosarcoma can be secondary to another disease, especially Paget's disease (8$32 \%$ of elderly osteosarcoma) or previous irradiation (3-8.5\% of elderly osteosarcoma) $[14,27,38,39]$. Only $3 \%$ of the total patients were recorded as having osteosarcoma in association with Paget's disease and $15.6 \%$ in association with previous radiotherapy. No association between secondary or primary osteosarcoma and survival was significant statistically; neither in the different studies nor in our study (Table 3). 
Metastases at the diagnosis have also been frequently observed in elderly patients, with an incidence between 5 and $33 \%$ and with a poor outcome [3,13,14,26-30]. This is confirmed in our study with $31.2 \%$ of the patients with systemic osteosarcoma at diagnosis and a significant poorer outcome with an overall survival of 2.8 years $(0.3-12.3)$ versus 4.2 years (0.6-12.6) for patients with a localised osteosarcoma $(\mathrm{p}=0.01)$.

In some studies, surgery type is correlated with the survival. Patients who underwent limbsalvage surgery have had a better survival than those with an amputation $[13,14,27,40]$. In our series, there was no significant difference between amputation and limb-salvage surgery but this can be biased by the histologically clean margin in all cases (Table 3 ).

The role of chemotherapy in elderly patients has remained controversial. Some reports stated that chemotherapy in the elderly is beneficial, while others did not. Antman et al.[41] reported that relatively lower effectiveness of chemotherapy was observed in adults with advanced disease. This may have arisen from two reasons: one is the biological feature of osteosarcomas in the elderly who have a lower sensitivity to standard chemotherapy than those in younger patients. The other reason is that chemotherapy was not given to patients who refused the treatment, to patients whose health conditions were not good enough to tolerate intensive chemotherapy.

According to the results of the present study and previous reports concerning osteosarcoma in the elderly, metastases at diagnosis and definitive surgery are critical factors for survival.

One of the main limitations of the present study is its retrospective nature. Precise analyses concerning the chemotherapy dose and intensity were lacking.

\section{Conclusion}

Our results described herein show that patients over the age of 50 years should be treated similarly to those in the younger age group with aggressive chemotherapy and complete surgical resection whenever it is possible. Prospective studies of treatment for patients in this age group are required to identify the most effective, tolerable chemotherapy. 
Figure 1: Kaplan-Meier survival curve for all patients (solid line is median survival, dotted lines represent upper and lower 95\% CI)

Figure 2: Kaplan-Meier survival curve for patients with a localised osteosarcoma at the diagnosis (solid line is median survival, dotted lines represent upper and lower 95\% CI)

Figure 3: Kaplan-Meier survival curve for patients with a systemic osteosarcoma at the diagnosis (solid line is median survival, dotted lines represent upper and lower 95\% CI)

Table 1: Description of the patients

Table 2: Histologic description of the osteosarcoma

Table 3: Survival of patients (one patient lost with a distal femur primitive systemic osteosarcoma)

\section{Acknowledgments: None \\ Conflict of interest: None}

\section{Bibliography}

[1] Dorfman HD, Czerniak B. Bone cancers. Cancer. 1995 Jan 1;75(1 Suppl):203-10.

[2] Okada K, Hasegawa T, Nishida J, Ogose A, Tajino T, Osanai T, et al. Osteosarcomas after the age of 50: a clinicopathologic study of 64 cases--an experience in northern Japan. Ann Surg Oncol. 2004 Nov;11(11):998-1004.

[3] Iwata S, Ishii T, Kawai A, Hiruma T, Yonemoto T, Kamoda H, et al. Prognostic factors in elderly osteosarcoma patients: a multi-institutional retrospective study of 86 cases. Ann Surg Oncol. 2014 Jan;21(1):263-8.

[4] Meyers PA, Gorlick R. Osteosarcoma. Pediatr Clin North Am. 1997 Aug;44(4):973-89.

[5] Picci P. Osteosarcoma (osteogenic sarcoma). Orphanet J Rare Dis. 2007;2:6.

[6] Mirabello L, Troisi RJ, Savage SA. Osteosarcoma incidence and survival rates from 1973 to 2004: data from the Surveillance, Epidemiology, and End Results Program. Cancer. 2009 Apr 1;115(7):1531-43.

[7] COVENTRY MB, DAHLIN DC. Osteogenic sarcoma; a critical analysis of 430 cases. J Bone Joint Surg Am. 1957 Jul;39-A(4):741-757, 757-8.

[8] Marcove RC, Miké V, Hajek JV, Levin AG, Hutter RV. Osteogenic sarcoma under the age of twenty-one. A review of one hundred and forty-five operative cases. J Bone Joint Surg Am. 1970 Apr;52(3):411-23. 
[9] Ottaviani G, Jaffe N. The epidemiology of osteosarcoma. Cancer Treat Res. 2009;152:3-13.

[10] Stark A, Kreicbergs A, Nilsonne U, Silfverswärd C. The age of osteosarcoma patients is increasing. An epidemiological study of osteosarcoma in Sweden 1971 to 1984. J Bone Joint Surg Br. 1990 Jan;72(1):89-93.

[11] Balducci L. Epidemiology of cancer and aging. J Oncol Manag. 2005;14(2):47-50.

[12] Bielack SS, Kempf-Bielack B, Delling G, Exner GU, Flege S, Helmke K, et al. Prognostic factors in high-grade osteosarcoma of the extremities or trunk: an analysis of 1,702 patients treated on neoadjuvant cooperative osteosarcoma study group protocols. $\mathrm{J}$ Clin Oncol. 2002 Feb 1;20(3):776-90.

[13] Harting MT, Lally KP, Andrassy RJ, Vaporciyan AA, Cox CS Jr, Hayes-Jordan A, et al. Age as a prognostic factor for patients with osteosarcoma: an analysis of 438 patients. $\mathbf{J}$ Cancer Res Clin Oncol. 2010 Apr;136(4):561-70.

[14] Grimer RJ, Cannon SR, Taminiau AM, Bielack S, Kempf-Bielack B, Windhager R, et al. Osteosarcoma over the age of forty. Eur J Cancer. 2003 Jan;39(2):157-63.

[15] Kager L, Zoubek A, Pötschger U, Kastner U, Flege S, Kempf-Bielack B, et al. Primary metastatic osteosarcoma: presentation and outcome of patients treated on neoadjuvant Cooperative Osteosarcoma Study Group protocols. J Clin Oncol. 2003 May 15;21(10):2011-8.

[16] Mankin HJ, Hornicek FJ, Rosenberg AE, Harmon DC, Gebhardt MC. Survival data for 648 patients with osteosarcoma treated at one institution. Clin Orthop Relat Res. 2004 Dec;(429):286-91.

[17] Lee JA, Kim MS, Kim DH, Lim JS, Park KD, Song WS, et al. Risk stratification based on the clinical factors at diagnosis is closely related to the survival of localized osteosarcoma. Pediatr Blood Cancer. 2009 Mar;52(3):340-5.

[18] Allison DC, Carney SC, Ahlmann ER, Hendifar A, Chawla S, Fedenko A, et al. A metaanalysis of osteosarcoma outcomes in the modern medical era. Sarcoma. 2012;2012:704872.

[19] Rosen G, Caparros B, Huvos AG, Kosloff C, Nirenberg A, Cacavio A, et al. Preoperative chemotherapy for osteogenic sarcoma: selection of postoperative adjuvant chemotherapy based on the response of the primary tumor to preoperative chemotherapy. Cancer. 1982 Mar 15;49(6):1221-30.

[20] Jaffe N, Carrasco H, Raymond K, Ayala A, Eftekhari F. Can cure in patients with osteosarcoma be achieved exclusively with chemotherapy and abrogation of surgery? Cancer. 2002 Nov 15;95(10):2202-10.

[21] Chou AJ, Geller DS, Gorlick R. Therapy for osteosarcoma: where do we go from here? Paediatr Drugs. 2008;10(5):315-27.

[22] Luetke A, Meyers PA, Lewis I, Juergens H. Osteosarcoma treatment - Where do we stand? A state of the art review. Cancer Treat Rev. 2014 May;40(4):523-32. 
[23] Ta HT, Dass CR, Choong PFM, Dunstan DE. Osteosarcoma treatment: state of the art. Cancer Metastasis Rev. 2009 Jun;28(1-2):247-63.

[24] Bacci G, Ferrari S, Longhi A, Picci P, Mercuri M, Alvegard TA, et al. High dose ifosfamide in combination with high dose methotrexate, adriamycin and cisplatin in the neoadjuvant treatment of extremity osteosarcoma: preliminary results of an Italian Sarcoma Group/Scandinavian Sarcoma Group pilot study. J Chemother. 2002 Apr;14(2):198-206.

[25] Bacci G, Briccoli A, Rocca M, Ferrari S, Donati D, Longhi A, et al. Neoadjuvant chemotherapy for osteosarcoma of the extremities with metastases at presentation: recent experience at the Rizzoli Institute in 57 patients treated with cisplatin, doxorubicin, and a high dose of methotrexate and ifosfamide. Ann Oncol. 2003 Jul;14(7):1126-34.

[26] Carsi B, Rock MG. Primary osteosarcoma in adults older than 40 years. Clin Orthop Relat Res. 2002 Apr;(397):53-61.

[27] Longhi A, Errani C, Gonzales-Arabio D, Ferrari C, Mercuri M. Osteosarcoma in Patients Older Than 65 Years. JCO. 2008 Nov 20;26(33):5368-73.

[28] Manoso MW, Healey JH, Boland PJ, Athanasian EA, Maki RG, Huvos AG, et al. De novo osteogenic sarcoma in patients older than forty: benefit of multimodality therapy. Clin Orthop Relat Res. 2005 Sep;438:110-5.

[29] Nishida Y, Isu K, Ueda T, Nishimoto Y, Tsuchiya H, Wada T, et al. Osteosarcoma in the elderly over 60 years: a multicenter study by the Japanese Musculoskeletal Oncology Group. J Surg Oncol. 2009 Jul 1;100(1):48-54.

[30] Bacci G, Ferrari S, Donati D, Longhi A, Bertoni F, Di Fiore M, et al. Neoadjuvant chemotherapy for osteosarcoma of the extremity in patients in the fourth and fifth decade of life. Oncol Rep. 1998 Oct;5(5):1259-63.

[31] Huvos AG. Osteogenic sarcoma of bones and soft tissues in older persons. A clinicopathologic analysis of 117 patients older than 60 years. Cancer. 1986 Apr 1;57(7):1442-9.

[32] Saifuddin A, Mitchell R, Burnett SJD, Sandison A, Pringle J a. S. Ultrasound-guided needle biopsy of primary bone tumours. Bone \& Joint Journal. 2000 Jan 1;82-B(1):504.

[33] Kissin MW, Fisher C, Carter RL, Horton LWL, Westbury G. Value of Tru-cut biopsy in the diagnosis of soft tissue tumours. Br J Surg. 1986 Sep 1;73(9):742-4.

[34] Klein MJ, Siegal GP. Osteosarcoma: anatomic and histologic variants. Am J Clin Pathol. 2006 Apr;125(4):555-81.

[35] Grimer RJ. Surgical options for children with osteosarcoma. Lancet Oncol. 2005 Feb;6(2):85-92.

[36] Song WS, Kong C-B, Jeon D-G, Cho WH, Kim MS, Lee JA, et al. Prognosis of extremity osteosarcoma in patients aged 40-60 years: A cohort/case controlled study at 
a single institute. European Journal of Surgical Oncology (EJSO). 2010 May;36(5):4838 .

[37] Hagleitner MM, Hoogerbrugge PM, van der Graaf WTA, Flucke U, Schreuder HWB, te Loo DMWM. Age as prognostic factor in patients with osteosarcoma. Bone. 2011 Dec;49(6):1173-7.

[38] Mankin HJ, Hornicek FJ. Paget's sarcoma: a historical and outcome review. Clin Orthop Relat Res. 2005 Sep;438:97-102.

[39] Bielack SS, Kempf-Bielack B, Heise U, Schwenzer D, Winkler K. Combined modality treatment for osteosarcoma occurring as a second malignant disease. Cooperative German-Austrian-Swiss Osteosarcoma Study Group. J Clin Oncol. 1999 Apr;17(4):1164.

[40] Tan P, Yong B, Wang J, Shen J, Huang G, Yin J, et al. Survival analysis of 311 patients with osteosarcoma of the extremities treated in a single institute. Chinese Journal of Orthopaedics. 2012;32(11):1032-9.

[41] Antman K, Crowley J, Balcerzak SP, Kempf RA, Weiss RB, Clamon GH, et al. A Southwest Oncology Group and Cancer and Leukemia Group B phase II study of doxorubicin, dacarbazine, ifosfamide, and mesna in adults with advanced osteosarcoma, Ewing's sarcoma, and rhabdomyosarcoma. Cancer. 1998 Apr 1;82(7):1288-95. 


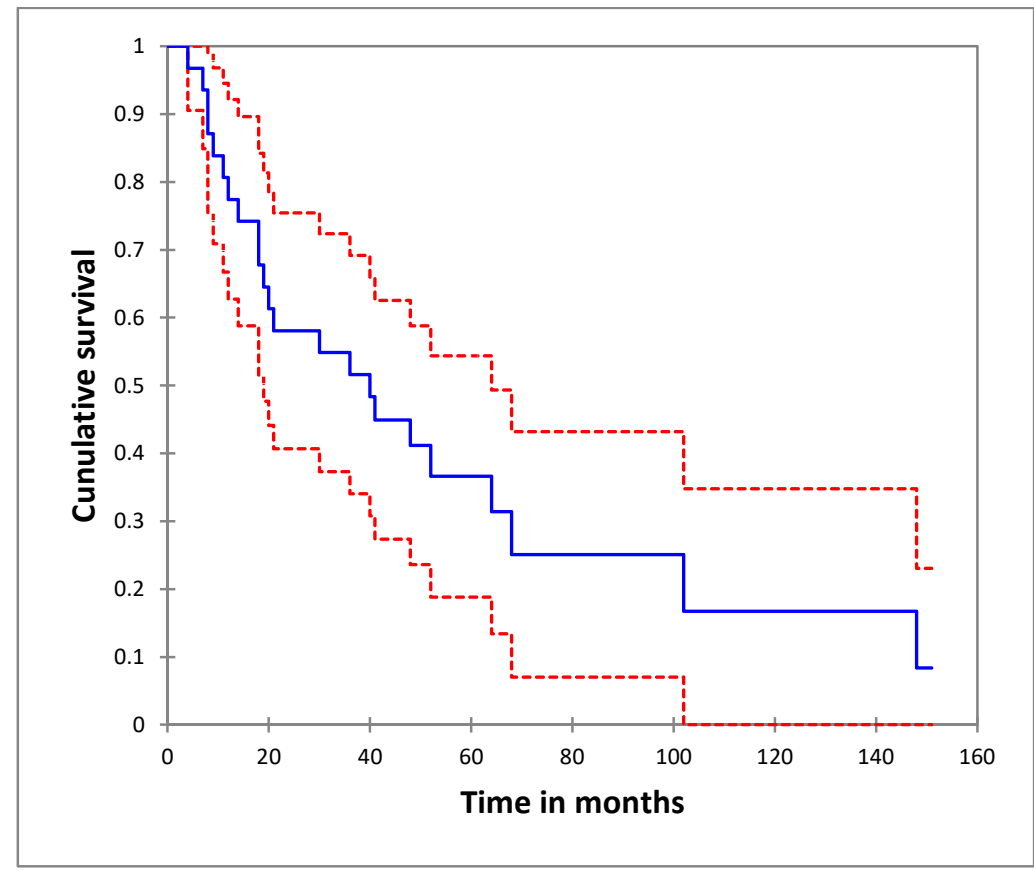

Figure 1: Kaplan-Meier survival curve for all patients (solid line is median survival, dotted lines represent upper and lower 95\% CI) 


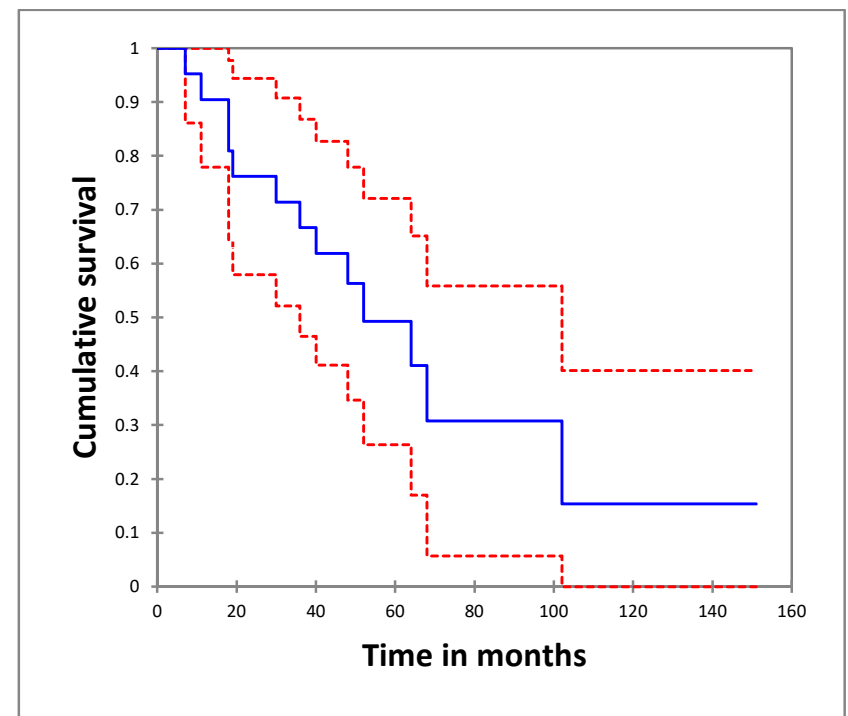

Figure 2: Kaplan-Meier survival curve for patients with a localised osteosarcoma at the diagnosis (solid line is median survival, dotted lines represent upper and lower 95\% CI) 


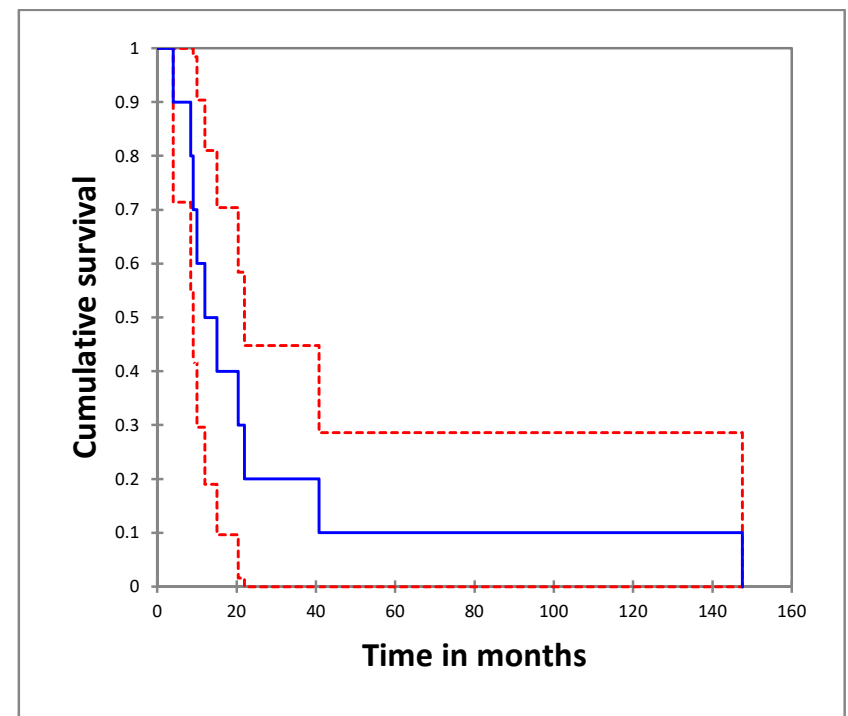

Figure 3: Kaplan-Meier survival curve for patients with a systemic osteosarcoma at the diagnosis (solid line is median survival, dotted lines represent upper and lower 95\% CI) 


\begin{tabular}{|c|c|c|c|c|}
\hline & & & 32 patients & $\%$ \\
\hline Mean ag & n-max) (yea & & $62.4(50-85)$ & \\
\hline Gender & Male & & 13 & 40 \\
\hline & Female & & 19 & 60 \\
\hline Site & Extremity & Distal femur & 10 & 31.3 \\
\hline & & Proximal femur & 6 & 18.8 \\
\hline & & Proximal humerus & 1 & 3.1 \\
\hline & & Diaphysis of femur & 1 & 3.1 \\
\hline & & Proximal tibia & 3 & 9.5 \\
\hline & & Distal tibia & 1 & 3.1 \\
\hline & & Others & 1 & 3.1 \\
\hline & Trunk & Pelvis & 5 & 15.6 \\
\hline & & Scapula & 2 & 6.2 \\
\hline & & Others & 2 & 6.2 \\
\hline Primary & condary & Primary & 26 & 91.2 \\
\hline & & Secondary & 6 & 18.8 \\
\hline
\end{tabular}

Table 1: Description of the patients 


\begin{tabular}{|l|l|c|c|}
\hline \multirow{3}{*}{ Broders' histology grade } & & 32 patients & $\%$ \\
\cline { 2 - 4 } & Grade III & 29 & 90.5 \\
\cline { 2 - 4 } Histologic type & Grade II & 3 & 9.5 \\
\cline { 2 - 4 } & Osteoblastic & 18 & 56.3 \\
\cline { 2 - 4 } & Fibroblastic & 7 & 21.8 \\
\cline { 2 - 4 } & Chondroblastic & 2 & 6.2 \\
\cline { 2 - 4 } & Telengiectasic & 2 & 6.2 \\
\cline { 2 - 4 } & Others & 3 & 9.5 \\
\hline
\end{tabular}

Table 2: Histologic description of the osteosarcoma 


\begin{tabular}{|c|c|c|c|c|}
\hline \multicolumn{2}{|l|}{ Variable } & \multirow{2}{*}{$\begin{array}{l}\mathrm{n} \\
22\end{array}$} & \multirow{2}{*}{$\begin{array}{l}\text { Median overall } \\
\text { survival (min- } \\
\text { max) (years) } \\
4(0.9-12.6)\end{array}$} & \multirow{3}{*}{$\begin{array}{l}\begin{array}{l}\text { Log-rank } \mathrm{p} \\
\text { value }\end{array} \\
0.02\end{array}$} \\
\hline \multirow[t]{2}{*}{ Site } & Extremities & & & \\
\hline & Trunk & 9 & $1(0.3-8.5)$ & \\
\hline \multirow[t]{2}{*}{ Type } & Primitive & 25 & $3.4(0.3-12.6)$ & \multirow[t]{2}{*}{0.28} \\
\hline & Secondary & 6 & $3.1(0.6-8.5)$ & \\
\hline \multirow[t]{2}{*}{ Initial metastasis } & Present & 9 & $2.4(0.3-12.3)$ & \multirow[t]{2}{*}{0.01} \\
\hline & None & 22 & $4.2(0.9-12.6)$ & \\
\hline \multirow[t]{2}{*}{ Surgery } & Present & 25 & $4.3(0.9-12.6)$ & \multirow[t]{2}{*}{0.0002} \\
\hline & None & 6 & $0.7(0.3-1.2)$ & \\
\hline \multirow[t]{2}{*}{ Surgery type } & Amputation & 5 & $3.2(1.5-4.3)$ & \multirow[t]{2}{*}{0.63} \\
\hline & Limb-salvage & 20 & $4.6(0.9-12.6)$ & \\
\hline \multirow[t]{2}{*}{ Chemotherapy } & Present & 20 & $4(0.3-12.6)$ & \multirow[t]{2}{*}{0.03} \\
\hline & None & 11 & $1.5(0.7-8.5)$ & \\
\hline \multirow[t]{2}{*}{ Treatment } & Chemotherapy+surgery & 19 & $4.3(0.9-12.6)$ & \multirow[t]{2}{*}{0.34} \\
\hline & Surgery alone & 6 & $3.5(1.2-8.5)$ & \\
\hline
\end{tabular}

Table 3: Survival of patients (one patient lost with a distal femur primitive systemic osteosarcoma) 